\title{
ICTIOFAUNA DE LA CUENCA DEL RIO TUMBES DPTO. TUMBES (PERU)
}

AMA MAC DONALD LOTFZ

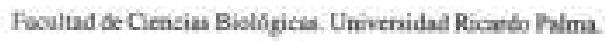

\begin{abstract}
RESUMEN
Se realiza un levantamiento de las especies fecicas propias del ro Tunbes, tanto especies endembess convo Chilobrycan deaterndan.

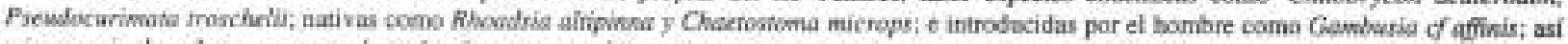

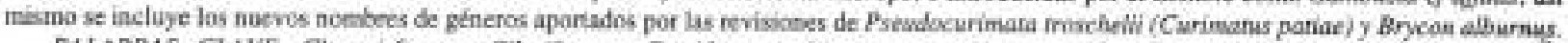

FALABRAS CLAVE: Characiffomes, Siluriformes, Perciformes, Cyprinobodoatriformes; sincnimia, maverial examinado, diagnosis, distribucion, no Tumbes.
\end{abstract}

\section{SUMMARY}

This work is a complished the suncy of ichthyic species,poper of the Tumbes river as moch as endenic species like Chilabrycon deaterodon,

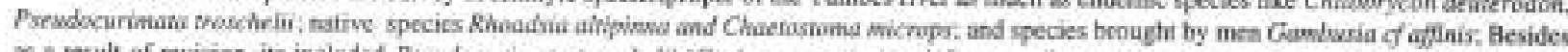

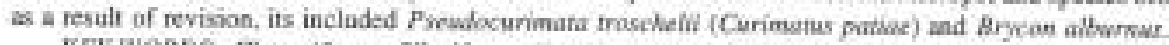
River

KEY WORDS: Characiforms, Silurifanms, Pensfoems, Cyprinodontifanms, Synonym, Examination Maverial, Diagnosia, Destribution, Tumbes

\section{INTRODUCCION}

El cauce del río Tumbes presenta una gran diversidad de especies ficticas, algunas consideradas endémicas y muchas de ellas propias de la zona norte del Perí. La presencia del hombre ha influido en la introducción de algunas especies favorables a la economía del poblador tumbesino, y que modifica de esta manera el contenido de la ictiofauna nativa, al competir por el habitat y la alimentación, así sc poede encontrar especies nuevas o introducidas en el río Tumbes.

La cuenca del rio Tumbes muestra varios cambios desde el represamiento de la margen derecha del río, realizada en la década pasada, ya que el río Tumbes provee de agua potable a los pobladores riberefos y es fuente de energía mecánica par la irrigación de cuitivos.

Se realiza el presente trabajo como un intento de realizar un levantamicnto de las especies feticas encontradas y aquellas que otras abundantes se ban tornado escasas o ausentes y se toma como referencia el trabajo de Chirichigno, 1963.

Paralelamente se incluye los nuevos nombres de géneros aportados por las revisiones de algunos grupos en particular, y las sinonimias.

\section{MATERIALES Y METODOS}

\section{Area de Estudio}

Corresponde al rio Tumbes que tiene $230 \mathrm{Km}$. de longitud y se ubica en el extremo noreste del Perú, departamento de Tumbes, aproximadamente $3^{\circ} 28^{\prime}$ y $4^{\circ} \mathrm{S}$ de latitud Sur. $80^{\circ} 30^{\prime}$ y $80^{\circ} 30^{\circ} \mathrm{W}$ meridiano de Gireenwich en la frontera entre Perú y Ecuador.

\section{Material Biológico}

Se analizaron 464 ejemplares del río Tumbes entre los ah̃os 1972 a 1988, colectados por Walter Gutierrez, Silvia Delgado, Hernán Ortega, Richard Vari, Patrick de Rham, Carlos Contreras e IMARPE.

\section{Métodos}

Las colecciones se realizaron en las siguientes estaciones:
I : SUR DE GARBANZAL.
Primera estación.
II : PUEBLO DE FRANCOS. Segunda estación.
III: RICA PLAYA.
Tercera estación.
IV: CAZADEROS: Cuarta estación.
$V$ : BOCANA MURCIELAGO. Quinta estación,

De las 18 especies encontradas, 10 no se encuentran en una estación determinada, debido a que en el moménto de la captura no se especificó la zona de procedencia a través del cauce del río Tumbes.

La identificación serealizó utilizando parametros tales como aspectos morfológicos (forma del cuerpo, forma de la cabeza, forma de los dientes, etc.), merísticos (números de radios, número de espinas, número de escamas, etc,) y datos morfométricos para lo cual se utilizó el Calibrador Vernier en mm. 
No se determinó el sexo de los individuos estudiados, aunque mostraban dimorfismo sexual evidente algunas especies.

Las observaciones se realizaron a simple vista y se utilizó el mieroscopio entereoscopio para observar estructuras pequef́as como dieates y escamas.

Para la categorización taxconómica se utilizaron los trabajos de EIGENMANN (1922), BOHLKE (1958), CHIRICHIGNO (1963), ORTEGA Y VARI (1986). Para los aspectos ecológicos se consultaron los trabajos de FRANK (1974), GERY (1977), MEDINA (1982) Y LOFTUS (1987).

En el material examinado se considera el número del lote, número de ejemplares, rango de la longitud estándar expresado en mm, la estación de colecta, en números romanos y fecha de colecta.

Ej. MUSM N ${ }^{\mathrm{a}} 2345,34$ (45.6-78.5)(III) 23_Ag.1991.

Los especimenes se encuentran en la Colección Ictiológica del Museo de Historia Natural de la UNMSM (MUSM) debidamente catalogados.

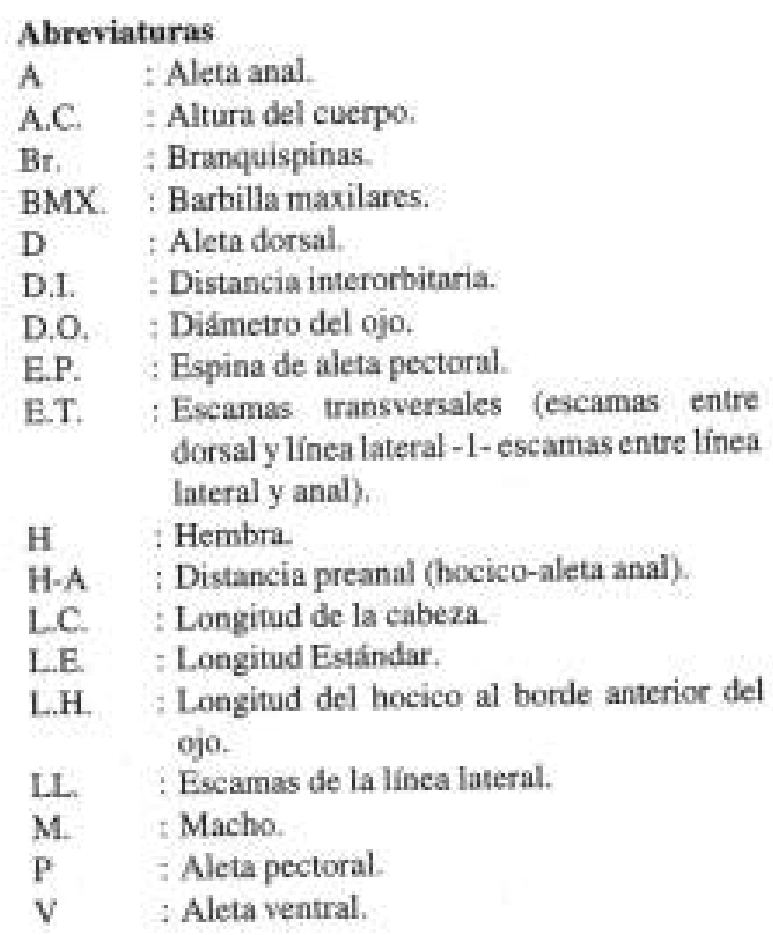

\section{RESULTADOS}

La ictiofauna analizada proviene de la Cuenca del rio Tumbes y está agrupada en 4 Ordenes, 11 familias, 14 géneros y 18 especies.

Una especie es endémica del rio Tumbes, Chïlobrycon deuzerodon, otra especie es introducida, Gambusia of affinis, y cuatro especies son de origen marino Mugil curema, Diapterus penivianus, Awaous transandeanus y Philypnus maculatus.

No se consideran especies colectadas en Puerto Pizarro. La composición de especies por estaciones sc manifiesta de la siguiente manera:

Tabla 1. Relación enfre la taxa y las cinco estaciones de colecta del rio Tumbes.

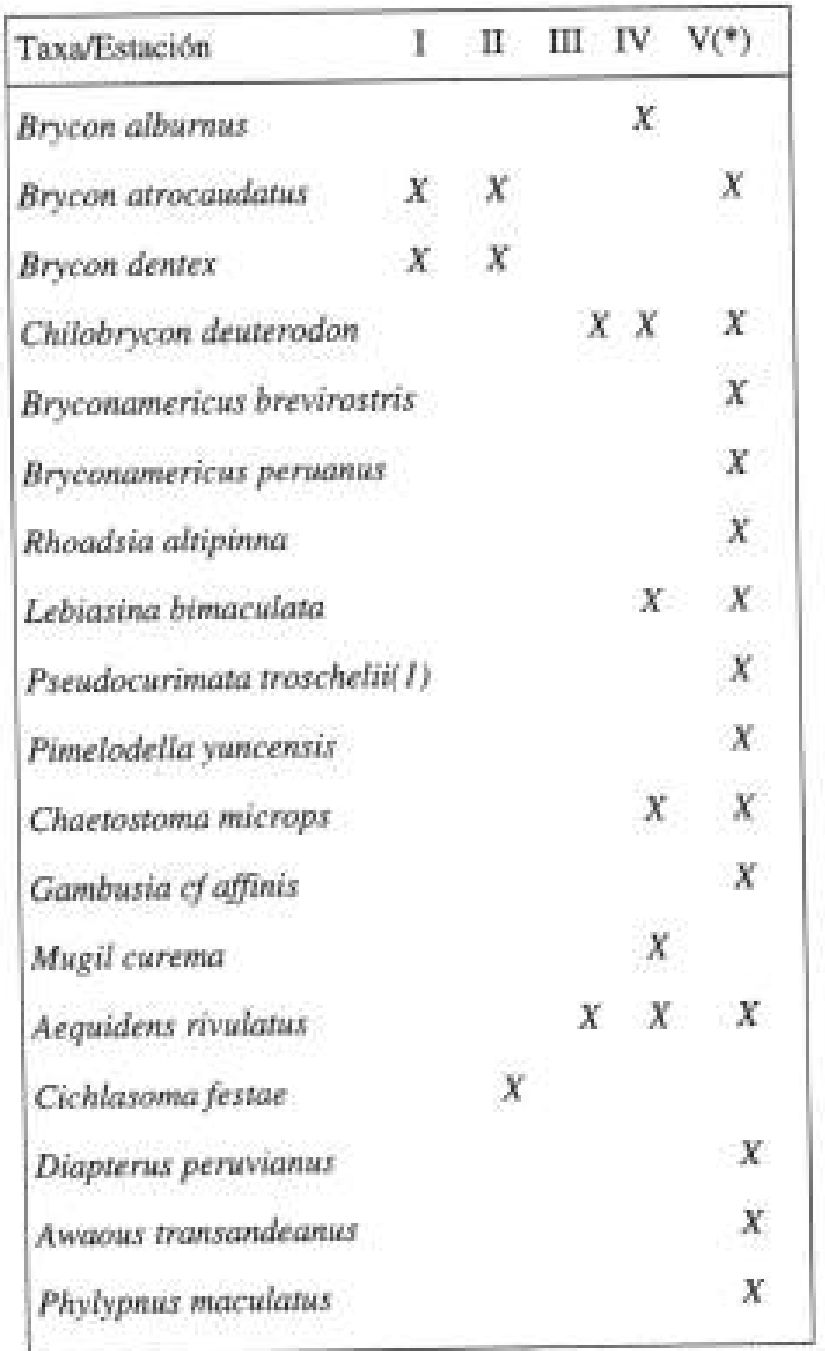

(1) Se toma como referencia Pozos Cajas (R. Zanumilla).

(*) No especifica la estación de colecta en la Cuenca del Rio Tumbes.

\section{PARTE TAXONÓMICA \\ CLASE OSTEICHTHYES \\ ORDEN : CHARACIFORMES \\ FAMIIIA : CHARACIDAE SUBFAMILIA : BRYCONINAE}

Brycon Muller \& Troschel, 1844.

Cuerpo alargado y moderadamente alto. Premaxilar con tres a cuatro series de dientes y dos en el dentario. Dientes cuspidados (Howes, 1982). 
Habitan ambientes lóticos y lénticos cerca a la superficie. Son omnivoros, prefieren alimentos vegetales, frutos, semillas, insectos (Ortópteros) y peces menores. Reproducción anual, durante épocas lluviosas (dic-mar). (Gery, 1977).

\section{Brycon alburnus (Günther, 1859)}

N. $V_{i}$ eDamas

\section{SINONIMIA}

Chalceas alburnus Ginther, 1859;149

Chalcinopsis albumus Guinthern, 1864:318. Gery, 1972: 931.

Bpycan acutus Bohlke, 1958:67. Myers \& Weitzmann, 1960:103. Gery, 1977:339. Gutiérrez et al; 1980. Tab. 4. Bncon alburnus Eigenmann \& Eigenmann, 1891:55. Eigenmann, 192:130. Gery, 1972:8. Ortega y Vari. 1986:7.

\section{MATERIAL EXAMINADO:}

MUSM N $^{\circ} 2603,1$ (170) (IV) 2Nov., 1977.

\section{DIAGNOSIS}

Dieates premaxilares en dos series, maxiliar poco prominente. A.C.: $2.9 \mathrm{en} \mathrm{L.E.,} \mathrm{escamas} \mathrm{pequeñas,} \mathrm{LL.} 47$.

\section{DISTRIBUCION}

Oeste de Ecuador (rio Esmeralda, Región de Guayas y Bahía de Guayaquil). Norte del Perú, Tumbes (rio Tumbes).

Brycon atrocaudatus (Kner \& Steindachner, 1863) N. $\mathrm{V}_{\text {: }}$ "Cascafe"

\section{SINONIMIA}

Chalceus atrocaudatus Kner \& Steindachner, 1863: 227. Brycon scapularis Fowler, 1911: 502. Myers \& Weitzmann, 1960:103.

Brycon ecuadoriensis Eigenmann \& Henn, 1917:687, Brycon atrocaudatus Günther, 1864:336. Starks, 1906:777. Bohlke, 1958:62. Chirichigno, 1963:20, Gery. 1977:335, Gutierrez 릐 all, 1980:7. Howes, 1982:9, Ortega y Vani, 1986:7. Sifuentes, 1990:27.

\section{MATERIAL EXAMINADO}

MUSM N' 2602, 8(79.5-107) 11 Nov., 1978.

MUSM N*2577, 28 (19.6-32) (1) 9.Ago., 1978.

MUSM N 1984, 1 (79.4) (II) 10. Ago, 1986

\section{DIAGNOSIS}

Dientes premaxilares en dos series, auxillar superior poco prominentes. A.C: 3.3 en L.E., escamas pequeñas. $L \mathrm{~L}: 54$. Sin banda lateral negra.

\section{DISTRIBUCION}

Vertientes Occidentales del Sur de Ecuador (Sistema de Guayas, róo Esmeraldas y río Marañón). Norte del Perú, Tumbes. (rio Tumbes y rio Zarumilla), Piura (Saña y Tinajones), Lambayeque (río Jequetepeque) y Ancash (río Santa).

Brycon dentex Günther, 1860

N.V. : weascafeo

\section{SINONIMLA}

Chalcinops dentex Günther, 1864:337

Brycon dentex Gūnther, 1860:240. Chirichigno, 1963:21. Gery, 1977:339. Gutiérrez es al, 1980: Tab. 4. Ortega y Vari, 1986:7.

\section{MATERIAL EXAMTNADO}

MUSM N 2601,16 (23.5-36.7) (I) 9 Ag, 1986

MUDM N $N^{\circ} 2576,23$ (6.79-18.6) (II) 10 Ago., 1986

\section{DIAGNOSIS}

Dientes premaxilares en tres series laterales, maxilar fuertemente proyectado. A.C.: 4.3 en L.E. Presencia de la banda lateral negra. Escamas grandes L.L: 39. Br. 19.

\section{DISTRIBUCION}

América Central. Vertientes. Vertientes Occidentales de Ecuador (rio Esmeraldas, nio Guayas). Norte del Perú, Tumbes (rio Tumbes).

Chïlobrycon Gery et de Rham, 1981

Cuerpo fusiforme, cabeza corta, hocico puntiagudo. Dientes del premaxilar en tres series, estapuladios, trilobados, aplanados y en poca cantidad sobre los maxilares. Ausencia del labio superior.

Prefieren los ambientes lóticos, cerca de la superficie, en las aguas claras. Se alimentan de vegetales, frutas y semillas. Reproducción desconocida (Gery et de Rham, 1981).

Chilobrycon deuterodo. Gery et de Rham, 1981.

N.V.: asabaloo

\section{SINONIMIA}

Chilabrycon deuterodom Gery et de Rham, 1981:7. Ortega y Vari, 1986:8.

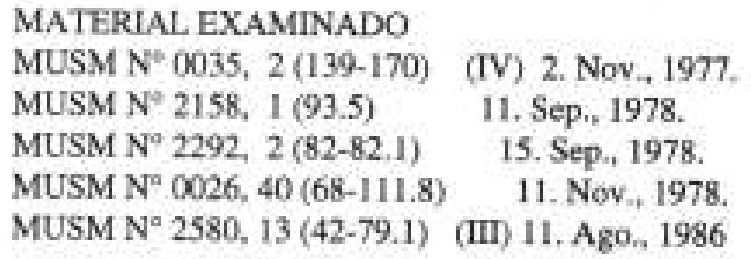

\section{DIAGNOSIS}

Cabeza conta, L.C: 3.6 en L.E., hocico bien puntiagudo debido a la ausencia del labio superior. L.H.: 4 en L.C., dientes premaxilares en 3 series, trilobados, aplanados, casi espatulados, en poca cantidad sobre los maxilares. Aleta pectoral en posición basal. Ausencia del lóbulo caudal medio, LL: 55. 


\section{DISTRIBUCION}

Vertientes del Pacífico de los Andes Ecuatoriales. Especic endémica de la zona norte del Perú, Tumbes (río Trapazol, pequet̂o afluente del rio Tumbes),

\section{SUB - FAMILIA TETRAGONOPTERINAE}

Bryconamericus Eigenmann, 1907.

Cuerpo alargado y pequeño, cabeza y parte anterior del tronoo gruesos. Line lateral completa. Premaxilar con dos dientes y una serie en el dentario.

Habitan aguas tranquilas en afluentes de ríos, en fondos pedregosos y ligeramente arenosos. Se alimentan de Plancton e insectos acuáticos. Reproducción anual. (Gery, 1977).

Bnconanericus brevirostris (Günther, 1859)

N.V.: aCarachitan

\section{SINONIMIA}

Chalceus brevirostris Günther, 1859:420.

Broconamericus brevirostris Eigenmann, 1922:151. Eigenmann, 1927:391. Chirichigno, 1963:23. Gery 1077:391. Gutiérrez st al, 1980: Tab. 4. Onega y Vari, 1986:7.

\section{MATERIAL EXAMINADO}

MUSM N $2582.30(24.4-67.8) \quad$ 10. Ag. 1986

\section{DIAGNOSIS}

Cuerpo pequeño, comprimidoy alargado. A: 1,39. LL: 43. Dos manchas humerales oscuras sobre la línea lateral. Radios caudales medios negros.

\section{DISTRIBUCION}

Vertientes Occidentales de Ecuador (río Vinces) y Norte del Perú, Tumbes (rio Tumbes y río Zarumilla).

Bryconamericus peraanus (Müller \& Troschel, 1945). N.V.: Carachitas

\section{SINONIMIA}

Tetragonoptenus peruanus Muller \& Troschel, 1845: 28. Tetragonopterus peravianus Gunther, 1864:327. Eigenmann \& Eigenmann, 1891: 53 .

Bryconamericus peruanus Eigenmann. 1910:434. Tortoneses, 1942:60. Chirichigno, 1963:22. Gery. 1977:390. Gutiérrezet al, 1980: Tab, 4, Gery et de Rham. 1981:10. Ontega y Vari, 1986:7. Sifuentes, 1990:29.

\section{MATERIAL EXAMINADO}

MUSM N $N^{*} 0147.5(81.5-89.6) \quad 27$, Oct., 1977.

MUSM N 2578, 1 (10.5) 5. Nov., 1977.

MUSN N ${ }^{\circ} 0166,18(49.5 \cdot 85) \quad 11$. Nov., $_{1978 .}$

\section{DIAGNOSIS}

Cuerpo pequeño y alargado. Dimorfismo sexual marcado, las hembras son notablemente más grandes que los machos. A:i,27. LL: 38. Mancha caudal contínua hasta la mitad de los radios caudales medios.

\section{DISTRIBUCION}

Vertientes Occidentales de Ecuador (río Peripa, río Zamora). Norte del Perú, Tumbes (río Tumbes y río Zarumilla), Lambayeque (río Reque), Ancash (rio Santa), Lima (Albufera «Medio Mundow, rio Rímac, Cañete).

\section{SUB-FAMILIA RHOADSINIINAE}

\section{Rhoadsia Fowler, 1911}

Cuerpo alto y comprimido. Aleta dorsal bien alta. Cuarto y quinto radios dorsales alcanzan a la aleta caudal. Línea lateral incompleta. Mancha negra caracteristica a los lados del cuerpo a la altura de la aleta dorsal. Los especimenes jóvenes y adultos presentan diferencias relacionadas a boca, dientes y maxilares, modificandose con la edad. Habitat restringido a tierras bajas, no observándose en cursos superiores. Se alimenta de semillas, insectos y crustáceos menores, Reproducción anual (Gery, 1977).

Rhoadsia altipinna Fowler, 1911

N.V.: adoradilla»

\section{SINONIMIA}

Rhoadsia altipinna Fowler, 1911:498. Gery, 1977:538, Gutiérrezet al, 1980: 11, Geryet de Rham, 1981:10 Ortega y Vari, $1986-9$.

\section{MATERIAL EXAMINADO \\ MUSM N ${ }^{\circ}$ 0027, 20 (45.2-88.3) 11.Noy., 1978}

\section{DIAGNOSIS}

Cuerpo alto y comprimido. A.C: 2.5 en L.E., los especimenes jóvenes y adultos presentan grandes diferencias relacionadas a boca, dientes y maxilar, modificándose con la edad. Aleta dorsal bien alta, cuarto y quinto radios dorsales alcanza a la aleta caudal. Línea lateral incompleta, oon perforaciones hasta la escama quince a dieciocho. LL: 39. Mancha característica a los lados del cuerpo a la altura de la aleta dorsall.

\section{DISTRIBUCION}

Costa Rica, Oeste de Colombia y Ecuador (río Chanchan), Norte del Perú, Tumbes (rio Tumbes).

\section{FAMIILA : LEBLASINIDAE SUB FAMILIA: LEBLASININAE}

Lebiasina Cuvier \& Valenciennes, 1846.

Cuerpo alargado, de sección casi cilíndrica. Sin aleta adiposa. Línea lateral hasta la región anterior de la aleta dorsal. Mancha en la base de los radios caudales medios y en la base de la aleta dorsal. Escamas grandes y oscuras. 
Se han adaptado a cursos de aguas muy irregulares, desde el nivel del mar hasta los $2,300 \mathrm{~m}$. Viven en corrientes fuertes o en charcas estancadas. Son predadores. Reproducción anual. (Gery, 1977).

Lebiasina bimaculata Valenciennes, 1846. N.V.: *Guavina»

\section{SINONIMIA}

Lebiasina bimaculata Valenciennes, en Cuvier \& Valenciennes, 1846;587. Günther, 1864:286, Morales Macedo, 1939:62. Fowler, 1940:752, 1945:132. Chirichigno, 1963:40. Gery, 1977:123. Gutiérrez et al, 1980: 11. Gery et de Rham, 1981:10. Ortega y Vari, 1986:10. Sifuentes, 1990:32.

\section{MATERIAL EXAMINADO \\ MUSM N ${ }^{\circ} 0352,2(41-60)$ (IV) 29. Oct. 1977. \\ MUSM N $N^{\circ} 0348,3(80-86.7) \quad$ 11.Nov.. 1978}

\section{DIAGNOSIS}

Cuerpo alargado de sección cilindrica, robusto y ágil. A.C.: Casi igual a L.C., L.C.: 3.9 en L.E., hocico poos prominente, L.H.: 4.6en L.C., D: i, 9, P:13. V:8. A:i,10. Escamas grandes y oscuras. Mancha en la aleta caudal y en la base de la eabeza. Con bada media longitudinal. LL:27.E.T: 7-1-6.

\section{DISTRIBUCION}

Vertientes del Océano Pacifico, vertientes occidentales del Ecuador y Perú, norte del Peru, Tumbes (río Tumbes yrío Zarumilla). Piura (río Pacasmayo y río Etén) Cajamarca (rio Paipay), Lambayeque (rio Jequetepeque y río Reque), La Libertad (río Chicama), Ancash (río Santa), Lima (Albufera *Medio Mundos, río Chillón, río Rimac, Puente Piedra, Chosica, Lurín).

\section{FAMILIA CURIMATIDAE SUB-FAMILIA CURIMATINAE}

\section{Pseudocarimata Fernández-Yepez, 19488}

Cuerpo alto y moderadamente alargado, sin dientes. labios encerrados en forma de ventosa. Perfil dorsal de la cabeza rocto. Ambientes lóticos. Son ilib́fagos, de reproducción anual (Vari, 1989).

\section{Pseudocurimata trosehelii (Günther, 1859)}

N.V.: «sábalo*

\section{SINONIMIA}

Anodus troschelit Ginther, 1859:418, Vari, 1989:2.

Curimatus troschelii Gunther, 1864:290. Endahl, 1937:5.

Pseudocurimata troschelii Fernández-Yépez, 1948:46, Vari, 1988:337., 1989:18.

Curimatus patiae Chirichigno, 1963:19. Gery, 1977:226. Vari y Ortega, 1986:11.
Cucrimata troschelii Gery, 1972:96.

\section{MATERIAL EXAMINADO}

MUSM N $256 \div 2$ 2(102.5-103.6)R. Zarumilia, Porns Cajas, 12.Ag, 1986.

\section{DIAGNOSIS}

Cuerpo alto y moderadamente alargado. A.C.: $2.9 \mathrm{en}$ L.C., cabeza puntiaguda, hocico subterminal. Mancha negra de forma triangular, horizontal y moderadamente alargada en el pedúnculo caudal. Ausencia de una mancha oscura a to largo de la superficie lateral y dorsal del cuerpo, asi como ea la base de los radios medios caudales. Escamas con poros a lo largo de toda la línea lateral.

\section{DISTRIBUCION}

Suroeste de Ecuador (río Suntiago y río Esmeraldas), sistema de ríos del Golfo de Guayaquil. Norte del Perú, Tumbes (rio Tumbes y río Faical).

\section{ORDEN SILURIFORMES \\ FAMILIA PIMELODIDAE}

\section{Pimelodella Eigenmann \& EIGENMANN, 1988.}

Primera aleta dorsal y pectoral espinosa. Procesos occipitales formando con la base de la dorsal un puente entre la cabeza y la aleta dorsal, una larga fontanela occipital estrecha. Aleta adiposa tan larga como la aleta anal. Espina pectoral tan corta como los radios. Aleta dorsal $y$ anal redondeadas.

Habitan generalmente ambientes lóticos. Se alimentan de insectos (Larvas y adultos), frutos y detritos, Su reproducción es anual. (Frank, 1974).

Pimelodella yuncensis Steindachner, 1912.

$\mathrm{N} . \mathrm{V}+$ aBagrea

\section{SINONIMIA:}

Pimelodella yuncensis Steindachner, 1912:135. Eigenmann, 1917:258. 1922:42. Morales. 1940:7. Fowler, 1945:43. Ortega y Vari, 1986:15.

Rhamdia gilli Starks, 1906:769, Morales Mucedo, 1940:76

\section{MATERIAL EXAMINADO}

MUSM N 2157, 1 (81) 11. Sep., 1978

MUSM N $3059,3(51-56.4)$ 18. Dic 1990

\section{DIAGNOSIS}

Procesos occipitales formando un puente con la base de la aleta dorsal, entre la cabeza y la aleta dorsal. Fontanela muy estrecha. Primer radio dorsal y pectoral espinoso. Aleta adiposa 3.4 en L.E., E.P.; 2.7 en L.E., aterturas branquiales amplias prolongándose hasta ta base de la aleta pectoral con ocho ganchos. Procesos humerales en forma de espinas. 


\section{DISTRIBUCION}

Noroeste del Perú. Norte del Perú, Tumbes (río Tumbes), La Libertad (rio Chicama y rio Moche), Piura, Sullana (río Etén).

\section{FAMILIA LORICARIIDAE SUB-FAMILIA ANCISTRIANE}

\section{Chaetostoma Von Tschudi, 1845}

Hocico ancho y redondeado, margen de la boca ancha, abultada y sin tentáculos. Escudo dorsal formadopor tres placas predorsales. Ausencia de procesos occipitales. base de la aleta dorsal formada por ocho placas. Escudos espiniscentes.

Habitan fondos pedregosos o debajo de piedras. Frecuentan aguas poco torrentosas. Se alimentan de algas. Presentan cortejo nupcial y cuidado de los hijos (Frank, 1974).

Chatostoma microps Günther, 1864

$\mathrm{N}, \mathrm{V}_{, ;} \approx$ Raspas

\section{SINONIMIA}

Chaetostoma microps Gunther, 1864:250. Steindachner, 1883:23, Fowler, 1954:150. Gutiértez et al, 1980:10. Ortega y Vari, 1986:17.

Hypostomus erinaceus (No Valenciennes) Günther, 1859:420.

MATERIAL EXAMINADO

MUSM N ${ }^{2} 2595,5(108-186.8)$ (V) 27. Ag., 1977.

MUSM N ${ }^{\circ} 0725,1$ (87) 11. Se, 1978

\section{DIAGNOSIS}

Escudo dorsal formado por tres placas predorsales. Ausencia de procesos humerales. Base de la aleta dorsal conformada por ocho placas. Todas las aletas fuertes preseatan el primer radio fuerte o espinoso. D:I,9, P:1,6. V: I,5. A: 1,4. Total de placas del tronco: 24. H-ano: $1.6 \mathrm{en} \mathrm{L.E.} \mathrm{Bmx:} 10$ en L.C.

\section{DISTRIBUCION}

Oeste de Ecuador (rio Totora, tío Zamora. Sistema de rfo Amazonas). Norte del Perú, Tumbes (rio Tumbes).

\section{ORDEN CYPRINODONTIFORMES FAMILIA POECILIDAE SUB-FAMILIA POECILIINAE TRIBU GAMBUSIINI}

Gambusia Baird, 1853

Cuerpos pequeño, con marcado diformismo sexual, siendo el macho y la hembra casi del mismo tamaño y con el mismo patrón de coloración. Aleta dorsal larga.
Habitan canales y charcas con densa vegetación. siendo de naturaleza agresiva y soportando pobres condiciones de oxigeno (Rosen, 1967).

Son abundantes y ubicables, se observan en pequefios grupos moviéndose constantemente próximos a la superficie, y convergen en un lugar en busca de alimento. Se alimentan de larvas de dipteros, se le considera un controlador biológico natural en la lucha antimosquitos.

Son vivíparos, con fecundación interns, pudiendo parir hasta seis veces al año en buenas condiciones. poniendo más de 150 alevines cada vez. La hembra requiere de una sola fecundación para producir puestas espaciadas. Los atevines se esconden entre las plantas para que no sean ingeridos por sus thadres (Luftus \& Kushan, 1987).

Gambusia of affinis (Baird \& Girard, 1853),

\section{SINONIMIA}

Heterandria affinis Baird \& Girard, 1953:390.

Gambusia affinis Girard, 1872.

\section{MATERIAL EXAMINADO}

MUSM N 1844,2 (H: $49.6 ; \mathrm{M}: 42.5)$ 6. Dic., 1988

\section{DIAGNOSIS}

Cuerpo pequeño, con marcado dimorfísmo sexual, tercer, cuarto y quinto radios anales del macho transformados en un organe copulador o gonopodio. El macbo es de forma alargada y la hembra redondeada. siendo ambos de igual longitud ( $\mathrm{H}: 49.6 ; \mathrm{M}: 42.5$ ), teniendo ambos sexos el mismo patrón de coloración. gris oscuro con cinco franjas transversales desde el dorso hasta la zona ventral del cuerpo.

\section{DISTRIBUCION}

Drenaje del Atlántico del Noroeste de U.S.A. rumbo al Sur a través de las Antillas Grandes hasta la Española y las Bahamas. Especie cosmopolita. Perú. Tumbes (rio Tumbes).

\section{ORDEN PERCIFORMES \\ SUB-ORDEN MUGILOIDEI \\ FAMILIA MUGILIDAE}

\section{Mugill Linaeus, 1758}

Cuerpo medianamente alargado, cabeza grande, boca ancha, oblicua, dientes solamente en las mandibulas, en una o dos series, escamas grandes y firmes. Primero dorsal con cuatro espinas fuertes, aleta anal con tres espinas, los jovenes con dos.

Peces cosmopolitas, de aguas tropicales y templadas. Su biotipo es Boca de Río, Orilla Marina (Medina, 1982). Remonta los ríos alejíndose de las 
desembocaduras; son catádromos, forman cardúmenes y son muy activos. Son euribalinos y viajan durante todo el anio buscando aguas de menor salinidad, son bentópelágicos, se encuentran cerca de fondos arenosos y arenofangosos próximes a la costa, en aguas turbias y limpias (Angell, 1968).

Son omnivoros, micrólages, su alimento proviene del fango rico en diatomeas llamándosele upez que como fango\%, para la digestión cuentan con un estómago con paredes musculares gruesas y un intestino largo que indica que la materia vegetal desempefia un papel muy importante en su dieta. Branquispinas filamentosas que le ayudan a extraer microalgas y otros materiales del sedimento por filtración. Desde aguas dulces los adultos regresan al mar para buscar desovaderos. No presentan un mes especial ya que es una forma continua. Se reproducen es pareja o en pequeñor grupos en aguas coesteras o estuario. Las larvas son pelágicas (Angell, op, cit).

Mugil curema Cuvier \& Valenciennes, 1836 N.V.: «Lisa plateada*

\section{SINONIMIA}

Mugil curema Cuvier \& Valenciennes. 1836:87. Eigenmann 1922:186, Moek \& Hildebrand, 1923:279. Chirichigno, 1963:35. Gutiérrezer al, 1980:11. Ortega y Vari, 1986:19.

Mugil ciliilabis Cuvier \& Valenciennes, 1836:151

Querimana harengus Jordan \& Jilbert. 1883:588. Eigentmann, 1922:187.

\section{MATERIAL EXAMINADO \\ MUSM N $1177,13(101-200) \quad 3$. Ene., 1974 \\ MUSM N=1175, $1(82.5) \quad 10$, Sep. 1978 \\ DIAGNOSIS}

Dos pequetias aletas dorsales bien separadas. D:IV. 1,8. Aletas ventrales de insercion abdominal. A:III, 9 en adultos y II, 10 en juvenules. Labio superior delgado, dientes secundarios simples y en fila uniseriada o dispuestos detrás de la fila principal y no presentes en la mandibula. Dientes villiformes, escamas grandes cicloideas en adultos. Sin línen lateral. L L:37.

\section{DISTRIBUCION}

Ambas costas de América. En las costas del Pacifico desde el Golfo de California (México) hasta Iquique (Chile). En el Atiantico desde Cabo Cope Cod hasta Brasil. Registrada también en Africa. En el Perú. Tumbes (Pto. Pizarro, río Tumbes, Zorritos). Trujillo (Pacasmayo).

\section{FAMILIA CICHLIDAE}

\section{Aequidens Eigenmann \& Bray}

Cuerpo moderadamente atto y comprimido, forma orbicular, los filamentos extremos de los radios de la aleta dorsal se extienden a lo largo del cuerpo hasta el final de la aleta caudal y los radios de la aleta anal solo un poco más cortos. Líncas horizontales en los lados del hocico, como carácter sexual secundario de los machos.

Coloridos, viven en ambientes lóticos y lénticos, en aguas blancas y turbias, variando de color con el habitat o elestado de ánimo. No hacen migraciones. Son pacificos y se encuentran en la vegetación filamentosa, Ocupan estratos intermedios y poco tormentosos del rio (Chang, 1995). Activos durante el día, son omnivoros. Oviparos, con fecundación externa, despoés de la postura el macho vigila los huevos y posteriormente los alevines, llegando incluso a transportarlos en su boca. El desove ocurre en cualquier época del afio. En época de reproducción son belicosos y exigen una demarcación territorial, además de buscar protección y alimento entre la vegetación filamentosa. La reproducción ocurre con facilidad en cautiverio, desovando sobre piedras y bojas.

Aequidens rivalanur (Güntber, 1859).

N. $V_{\text {i }}$ mojarras

\section{SINONIMLA}

Chromis rivalatur Guinther, 1859:418.

Acara rivalata Boulenger, 1899:5

Acara pulchra (No Gill) Günther, 1862:280.

Aequidens azurifera Fowler, 1911:515.

Aequidens rivulatus Starks, 1906:797. Evermann \& Radeliffe, 1917:116. Eigenmann, 1922:199, Chirichigno, 1963:60, Gutiérrez ss al. 1980-11. Ortega y Vari, 1986:20. Sifuentes, 1990:53.

\section{MATERIAL EXAMINADO}

MUSM N" 1022, 1 (60.2) 8. Mar., 1972.

MUSM N 1235, 11 (47.8-114) (V) 27, Oet, 1977.

MUSM N $1051,8(51-112.6)$ (IV) 30. Oct, 1977.

MUSM N 2590,42 (38.8-105) 11. Nov, 1978

MUSM N=1089, 2 (71.6-90.2) 21. Oct., 1986

DLAGNOSIS

Cuerpo moderadamente alto y comprimido, forma orbicular. A.C: 2.3 en L.E., L.C. 2.6 en L.E., los filamentos extremos de los radios de la aleta poca más cortos, D: XIV, 11. A: III, 8.

\section{DISTRIBUCION}

América del Centro y del Sur. Ecuador. Ica, Vertientes Occidentales de Ecuador y Perú, Tumbes (rio Tumbes). Ancash (rio Santa), Piura (Pacusmayo).

\section{Cichlasoma Swaison, 1839}

Cuerpo çmprounido, con una pequeña joroba, perfil dorsal convexo, cuerpo ovalado, labios gruesos, escamas largass, las espinas de la aleta anal $50 n$ de tres a seis, presenta una mancha negra posterosuperior en la mejilla. Presenta una mancha medio lateral. 
Viven en aguas lénticas y lóticas con vegetación fluctuantes donde buscan protección y alimento. Son diurnos. Cambian de color súbitamente por efecto de la intensidad de la luz o cuando se les molesta según el estado de ánimo (Campos, 1986).

Se alimenta de algas, larvas de insectos, crustáceos, pequefios peces, detritus vegetal y sedimento. El intestino presenta una longitud media de 0.7 veces la longitud total lo que refuerza su característica de omnívoro (Campos, op. cit).

Son ovíparos, con fecundación externa, en época de desove son belícosos e insociables, generalmente la hembra coloca los óvulos sobre una superficie previamente limpiada y el macho lo fecunda. Presenta de tres mil a cuatro mil huevos grandes de $1.2 \mathrm{~mm}$.

En su ambiente natural se reproducen cuando las aguas tienen temperaturas elevadas (diciembre-marzo) y en cautividad alcanzan la madurez sexual rápidamente (tres a cuatro meses). El desove se lleva a cabo en sustrato firme como troncos o piedras y es necesario el establecimiento del territorio, no necesita vegetación ya que la pareja de cria los destroza al cavar constantemente en la arena y presenta cuidado por los huevos, larvas y alevines (Buchup, 1985)

Cichlasoma festae (Boulenger, 1899)

N.V: $\propto$ Mojarra

\section{SINONIMIA}

Heros festae Boulenger, 1899:6, Pellegrin, 1900:230.

Cichlasama festae Eigenmann, 1922:206, Chirichigno, 1963:61. Gutiérrez et al, 1980. Tabla 4, Ontega y Vari, 1986:20

\section{MATERIAL EXAMINADO}

MUSM N $0833,6(70-114.1$ (III) 15. Sep., 1978

\section{DLAGNOSIS}

Cuerpo comprimido, de forma orbicular, con una pequeña joroba. A.C.: 2.1 en L.E., L.C.: 2.5 en L.E. D.O.: 4.1 en L.C., D. I: 3.1 en L.C., Hocico en punta. L.H.: 2.3 en L.C. D: XIV, 12, A: V,8. Espina parhipural corta o asuente. Línea lateral dividida en dos a la altura de la aleta dorsal blanda. Escamas grandes, largas y ctenoideas.

\section{DISTRIBUCION}

Ecuador, (Sistema del rio Guayas), Norte del Perú, Tumbes (rio Tumbes).

\section{FAMILLA GERREIDAE}

\section{Diapterus Cuvier, 1830}

Cuerpo corto, fuertemente comprimido. Línea lateral completa. Premaxilares protractiles. Espinas de la aleta dorsal fuertes, aleta anal con tres espinas siendo la segunda muy fuerte. Eurihalinos, presente en el biotipo: Orilla marina, Manglar, Boca de Rio (Medina, 1982).

Son omnivoros, se alimentan de animales bentónicos como anélidos, crustáceos, moluscos, cerdas de poliquetos, helmintos, filamentos de algas, cnidarios, considerándolos micrófagos, obteniendo su alimento por filtración de sedimento (Hildebrand, 1946).

Diapterus perwvianus (Cuvier, 1830)

N.V.: *Periche*

\section{SINONIMIA}

Guerres peruvianus Cuvier, 1830:467. Evermann \& Radcliffe, 1917:92.

Diapterus penwianus Tortonese, 1939:128, Hildebrand, 1946:43, Chirichigno, 1963:52. Gutierrez ef al, 1980: Tabla 4. Ortega y Vari, 1986:2.

\section{MATERIAL EXAMINADO}

MUSM N $N^{\circ} 2579,2$ (53.8-56.4) 13. Nov., 1977

\section{DIAGNOSIS}

Cuerpo corto, fuertemente compromido y alto. A.C. 2.2 en L.E., L.C.: 2.7 en L.E., Boca Protusible. L.H.: 2.5 en L.C., preoperculo distinguiblemente aserrado. D.IX,9 y IX,10, P:13. V: I,5. A: III,9. Escamas pequeñas en la parte superior y presenta huesos como crestas. Borde del preorbital suave.

\section{DISTRIBUCION}

Desde Mazatlán (México), hasta el Callao (Perú), Mazatián, Guatemala, Panamá, Ecuador y Perú.

Perú, Tumbes (río Tumbes), Paita, Capón y el Callac.

\section{SUB-ORDEN GOBIOIDEI FAMILIA ELEOTRIDIDAE}

\section{Philypnus Evermann \& Radeliffe, 1917}

Aletas ventrales separadas sin formar una ventosa ventral. Cuerpo alargado, cabeza desnuda anteriormente a la región interorbitaria, aberturas branquiales se extienden hacia adelante por debajo de los ojos. Aleta dorsal coa seis espinas.

Son bentónicos, en el día se esconden entre las rocas, ocultos en la vegetación acuática. Son nocturnos, eurihalinos, viven en las costas, esteros, a nivel del mar hasta $115 \mathrm{~m}$. Su régimen alimenticio consta de organismos bentónicos como gusanos, crustáceos, moluscos y peces pequeños. Sus crias son abundantes cerca a la costa (Bussing, 1987).

Philypnus maculatus (Günther, 1859)

N.V:; Guavina*

\section{SINONIMIA}

Lembus macularus Günther, 1859: 505 
Philypnus laterous Starks, 1906:799.

Philypnus maculatus Evermann \& Radcliffe, 1917:133. Eigenmann, 1922:210, Chirichigno, 1963:6, Gutierrez et. al. 1980: Tabla 4. Ortega y Vari, 1986:21.

\section{MATERIAL EXAMINADO}

$\begin{array}{lll}\text { MUSM N 2574, } 1(99.7) & \text { 13. Nov. } 1977\end{array}$

MUSM N $N^{\circ} 2584,17(43.7-103.4)$ 10. Agos., 1986

\section{DIAGNOSIS}

Aletas ventrales separadas sin formar una ventosa ventral. Preopérculo no aserrado. Vómer con dientes villiformes, las aberturas branquiales se extienden hacia adelante por debajo de los ojos. Aleta anal sin espinas. Escamas ctenoideas pequenias. LL: 59.

\section{DISTRIBUCION}

Vertientes occidentales del Océano Pacifico, desde Baja California hasta Perí. Méxjeo (rio Yaque), Panamá, Ecuador. Perú, Tumbes (rio Tumbes). Piura (rio Jequetepeque).

\section{FAMILIA GOBIIDAE}

\section{Awaous}

Cuerpo alargado, aletas ventrales unidas formando una ventosa, ovalada, más larga que ancha. Forma giobosa y prominente de los ojos. Dientes cónicos en filas, branquispiras preséntes. Frecuentemente estân adheridos por sus ventosas a troncos y piedras. Son eurihalinos y catádromos aunque son marinos viven en aguas salobres de estuario o aguas continentales. Prefieren fondos de arena a $25-31^{\circ} \mathrm{C}$, se les encuentra desde aguas estancadas hasta desembocaduras de rios y hasta 120 m.s.n.m.

Son voraces, e ingieren gran cantidad de detritus. microvivalvos y algas filamentosas, siendo omnivoros. Se reproducen cerca del río pero antes el macho prepara el nido bajo tierra. Los huevos son piriformes adhesivos. Las Jarvas son pelágicas (Bussing, 1987).

Awaous transandeanus (G0nther, 1861)

N.V.: *Chupapiedras*

\section{SINONIMIA}

Gobius transandeanus Guinther, 1861:62.

Awaows fransandeanus Regan, 1913:473. Meek \& Hildebrand, 1916:368. Eigenmann, 1922:216, Chirichigno, 1963:69. Gutiérrez et al, 1980. Tabla 4. Ortega y Vari, 1986:21.

\section{MATERIAL EXAMINADO}

MUSM N 2575, 1 (99) 113. Nov.. 1977.

\section{DIAGNOSIS}

Aletas ventrales unidas, formando una ventosa ventral. Cabeza desnuda. L.C.; 3,3 en L.E., interorbital no elevado hacia adelante, D.1.: 5.4 en L.C., forma globosa de ojos. D.0.; 6.0 en L.C., sin línca lateral.

\section{DISTRIBUCION}

Vertientes Occidentales del Sur de México, Oeste de Ecuador, desde Somira (México) hasta el norte del Perú, Tumbes (rio Tumbes).

\section{DISCUSION}

- Pseudocarimata troschelli se encuentra en discusión, si es la misma especie de $P$. boulenger, $P$. penaanas o es una nueva especie, dado el endemismo que presentan los curimátidos a consecuencia de procesos históricos, ecológicos, geológicos, climatológicos y biológicos. Presentan un gran número de especies, como producto de la evolución de millones de años de la existencia de dos a trescientas especies, to que Gery llama «especiación explosivas. Lo que ocurrió en Tumbes fue probablemente, que estás especies se aislaron con la formación de la cordillera de los Andes a partir de un antecesor común, evolucionaron a formas actuales de manera que se encuentran intimamente relacionadas unas con otras habiendo desarrollado características diferentes en cada zona de evolución. (Eigenmann, 1910).

-P. de Rham, 1990, publicó sus observaciones en la costa del Perú y determinó dos tipos de Aequidens rivulatus, uno agoldens en la zona norte del Perí con características de color: amarillo naranja predominante, $y$ otro usilver $* \mathrm{en}$ la costa de Lima de características de color: azul iridiscente predominante. Opino que proponer diferencias definitivas a partir de un patrón de coloración no es suficiente y se hace necesario utilizar ctros métodos tales como número de vértebras, nichos ecológicos difereaciables, etc, para tener un sustento evolutivo y una hipotesis filigenéticat que los diferencie.

- El trabajo de Chirichigno (1963), presenta numerosas especies de onigen marino para la zona de esteros, faltando varios characiformes propios de la cuenca del río Tumbes como Chilobrycon deuteradon y Rhoadsia altipina, asi como el Loticaridae Chaetostoma microps que tiene valor económico.

- En el trabajo de Gutierrez (1980), se mantiene audn algunos nombres antiguos, tales como Curimatus patiae en lugar de Pseadocurimata troschelli y tampoco considera a Chilobrycon deuterodon, especie endémica del ró Tumbes.

- Bnyean albamas sc cita en este trabajo después de considerarlo como Brycon acutus en Bohlike (1958) y Gery (1977).

- Chilobrycon deuterodon recien se inchuye en el catálogo como pez endémico de la zona norte del Perú. 
- Pimelodella yuncensis amplia su área de distribución que antiguamente era solamente hasta el Rfo Moche en Piura (Dávila, 1973).

- Gambusia cf affinis se considera un pez introducido que habita las zonas de canales de marea y pozos de langostinos en gran cantidad.

\section{REFERENCIAS BIBLIOGRAFICAS}

ANGELL. L.O. 1986. Algunos Aspectos de la Biologia de la LisaMugil curema Valenciennes, en aguas Hipersalinas del Nororiente de Venezuela EDIMAR, 51:223-238.

BOHLKE, E.J. 1958. Studies on Fishes of the Family Characidae. No 14 A. Report on Several, Extensive Recent Collector from Ecuador. Proc. Acad. Nat. Sci. Phil. IIO (14): 1- 21.

BUSSING, W.A. 1987. Peces de las Aguas Continentales de Costa Rica. Ed. Universidad Costa Rica.

CAMPOS, A.J. 1986. El Recurso Pesquero de Guapote (Cichlasoma dovii) en el embalse del Arenal Costa Rica. Contr. No. 105. CIMAR. Revista de Biologia Tropical 43: 215-219.

CHANG, F.I. 1995. Diversidad y Estructura de las comunidades de peces del río Tumbes - Perú: Tesis para optar Título Profesional de Licenciado en Biología. Universidad Ricardo Palma. Lima - Peni.

CHIRICHIGNO, N. 1963. Estudio de la Fauna Ictiológica de los Esteros y parte baja de los ríos del Dio. de Tumbes (Perú). Serv, Div. Sci. 22: 1-87.

CHIRICHIGNO, N. 1974. Clave para Identificar los Peces Marinos del Perú. Instituto del Mar del Perú. Informe No. 44, 387 pp.

EIGENMANN. H.C. 1910. The Origen of the Fish-fauna of the Fresh waters of South America.
EIGENMENN, H.C, 1922. The Fishes of Western South America. Part I : :9, 1:1-344, PT:1-38.

FRANK, S. 1974. Gran Enciclopedia Hustrada de Peces. Ed. Lectura.

GERY, J, 1977, Characoids of the World. T.F.H. Pub. Neptuno; 391.

GERY, J. \& P. de RHAM. 1981. Un nouveau poisson Characide endemique du río Tumbes au Nord du Perou. Chilobrycon deuteradon n.g.sp. (Characoidei). Rev. Francaise d' Aquariologie. 8 (I) 1-12.

HILDEBRAND, S.F. 1946. A Descriptive Catalog of the Shore Fishes of Peru. Bill. U.S.A. Natural Museum, 189: 1530 .

HOWES, G. 1982. Review of the Genus Brycon (Teleostei: Characoidei). Bull. Britain Museum Narumal History. ZooL.,43:9

LOFTUS, W \& J.A KUSHLAN. 1987, Freshwater Fishes of Southern, Florida. Bull. Florida State Museum Biology Science, $3 I: 149-343$.

MEDINA CH, W, 1982. Ecoanalisis de los Peces Osteichthyes Comunes de las Aguas Costeras del Peró según la forma de Dentición, Biotopo, obtención del Alimento. Revista Peruana de Biología 2; 77-133.

ORTEGA, H. \& R.P. VARI. 1986. Annotted Checklist of the Fresh Water Fishes of Peru. Smith. Contr. Zool No, 437: 1-26.

RHAM de 1990. Poissons de la cota du Perou. Revue Aquariophile Bimestrille Aquarama, 24; 13-20

ROSEN, D.E. 1967. New Poeciliid Fishes of Some South and Central American Formes. Amer, Museum Novitates. 2303.

VARI, R.P. 1989. Systematics of the Neotropical Characiforms Genus Pseudocurimata Fernández-Yapez (Pisces: Ostariophysis). Smith. Contr. Zool. Na. 498: 1-28. 